\title{
A Double Category Theoretic Analysis of Graded Linear Exponential Comonads
}

\author{
Shin-ya Katsumata $\left.{ }^{(}\right)$ \\ National Institute of Informatics, Tokyo, Japan \\ s-katsumata@nii.ac.jp
}

\begin{abstract}
Graded linear exponential comonads are an extension of linear exponential comonads wih grading, and provide a categorical semantics of resource-sensitive exponential modality in linear logic. In this paper, we propose a concise double-category theoretic formulation of graded linear exponential comonads as a kind of monoid homomorphisms from the multiplicative monoids of semirings to the composition monoids of symmetric monoidal endofunctors. We also exploit this formulation to derive the category of graded comonoid-coalgebras, which decompose graded linear exponential comonads into symmetric monoidal adjunctions plus twists.
\end{abstract}

\section{Introduction}

One of the important discoveries in substructural logic is the decomposition of the intuitionistic implication $\phi \Rightarrow \psi$ using the linear implication $\multimap$ and the exponential modality!. This discovery was studied by Girard through his linear logic, which brought many new ideas and perspectives to logic and programming language semantics.

Inside linear logic proofs, propositions with the exponential modality ! $\phi$ can be freely copied or discarded. Later, it was realized that by adding a copy limit to the exponential modality, like $!_{r} \phi$, linear logic gains fine control of assumption usage. This idea was first implemented in bounded linear logic [9], and studied in connection with implicit complexity theory $[4,14]$. Indexed exponential modalities $!_{r}$ were then used in wider context: resource management in programming languages $[3,7,8,20,23]$ and control of sensitivity in the metric semantics of programs $[5,21]$.

The categorical structure corresponding to the exponential modality ! was studied by various researchers, and it was identified as a categorical structure called linear exponential comonad [1]. One of the celebrated results about linear exponential comonads is that any symmetric lax monoidal adjunction:

$(\mathbb{D}, 1, \times) \frac{L}{<\frac{\perp}{R}}(\mathbb{C}, \mathbf{I}, \otimes) \quad$ (the monoidal structure $1, \times$ is cartesian)

yields a linear exponential comonad $L \circ R$, and every linear exponential comonad $D$ arises in this way - for $\mathbb{D}$ take the category of Eilenberg-Moore coalgebras of $D$.

(C) The Author(s) 2018

C. Baier and U. Dal Lago (Eds.): FOSSACS 2018, LNCS 10803, pp. 110-127, 2018.

https://doi.org/10.1007/978-3-319-89366-2_6 
The categorical structure corresponding to the indexed exponential modality $!_{r}$ has been proposed as exponential action [3] and graded linear exponential comonad [7]; they are two different presentations of the same data. Compared to linear exponential comonads, however, categorical understanding of graded linear exponential comonads is not well-established. The aim of this paper is to contribute to this point. Concretely speaking, we show the following categorical results about graded linear exponential comonads:

- We give a new concise formulation of graded linear exponential comonads as vertical monoid homomorphisms from multiplicative monoids of semirings to the composition monoids of symmetric lax monoidal endofunctors. This formulation is given in a rather complex multi-double category of symmetric monoidal categories. The slogan is "to represent a complex structure in a simple category as a simple structure in a complex category".

- In the multi-double category, vertical monoid homomorphisms themselves can be seen as monoids. By considering actions of such monoids, we obtain the concept of graded comonoid-coalgebras. They are an extension of EilenbergMoore coalgebras to graded linear exponential comonads, and the category of graded comonoid-coalgebras provides a resolution of graded linear exponential comonads by a symmetric lax monoidal adjunction plus a twist.

\section{Related Work}

Graded linear exponential comonads were first introduced as exponential actions in [3], and an equivalent definition was given in [7]. This paper adopts the latter definition as the starting point of study. These papers also consider linear type systems with an indexed exponential modality $!_{r} \phi$, which is directly interpreted by a graded linear exponential comonad. This paper, however, focuses only on the categorical axiomatics of the indexed exponential modality, and omit its syntactic theory. In [2], Breuvart and Pagani gave a construction of graded linear exponential comonads from a set of data called stratification. They derived various graded linear exponential comonads on the category of sets and binary relations and the category of coherence spaces. Structures close to, but different from, graded linear exponential comonads were considered in the categorical semantics of the following calculi: INTML for interactive computation [23], coeffect calculus [20] and bounded affine types system [8].

Looking at the dual structure, graded monads, first considered in mathematics $[6,25]$, were recently used in the semantic study of logic, systems and programming languages $[13,18,19,22]$. The resolution of graded monads were studied in [12], mildly extending a classic work by Street [26]. The major difference between graded monads and graded linear exponential comonads is the way how they interact with the monoidal structure. In [13] only strengths were considered for graded monads, while graded linear exponential comonads interact with monoidal structures in an intricate manner.

The multicategory of symmetric lax monoidal multifunctors is related to the 2-multicategory of $T$-algebras for a pseudo-commutative 2-monad $T$ [11]. 
Hyland and Power studied multifunctors that are symmetric strong monoidal in each argument, while in this paper we weaken "strong" to "lax". Yet, we think that by suitably extending their theory, the symmetric lax monoidal multifunctors can also be given in the language of 2-monad theory.

Monoids in the multicategory $\mathbf{M S M C}$ in Sect. 5 are similar to the distributivity studied in [15], where Laplaza considered two symmetric non-strict monoidal structures together with a colax distributivity between them. On the other hand, in this paper, we consider a strict monoidal structure on top of the underlying symmetric (non-strict) monoidal structure, and a lax distributivity between them.

\section{Preliminaries}

For symmetric monoidal categories and symmetric lax monoidal functors, see [16]. In a symmetric monoidal category $\mathbb{C}$, by $\iota: \mathbf{I} \otimes \mathbf{I} \rightarrow \mathbf{I}$ we mean the isomorphism $\lambda_{\mathbf{I}}=\rho_{\mathbf{I}}$, and by $\tau:(A \otimes B) \otimes(C \otimes D) \rightarrow(A \otimes C) \otimes(B \otimes D)$ we mean the symmetry swapping the second and third component of the tensor product. For functors $F_{i}: \prod_{j=1}^{m_{i}} \mathbb{C}_{i, j} \rightarrow \mathbb{D}_{i}$ where $1 \leq i \leq n$, we define $F_{1} \times \cdots \times F_{n}$ to be the composite functor $\prod_{1 \leq i \leq n, 1 \leq j \leq m_{i}} \mathbb{C}_{i, j} \rightarrow \prod_{i=1}^{n}\left(\prod_{j=1}^{m_{i}} \mathbb{C}_{i, j}\right) \rightarrow \prod_{i=1}^{n} \mathbb{D}_{i}$, whose codomain is the product category without the nesting of products.

\section{Graded Linear Exponential Comonad}

In this paper, comonads are graded by a partially ordered semiring. It is a tuple $(R, \leq, 0,+, 1, *)$ such that $(R, 0,+, 1, *)$ is a unital semiring (not necessarily commutative) and,$+ *$ are monotone in each argument w.r.t. the partial order $\leq$. The partially ordered monoids of additive and multiplicative parts of $R$ are denoted by $R^{+}=(R, \leq, 0,+)$ and $R^{*}=(R, \leq, 1, *)$, respectively.

Let $\mathbb{C}, \mathbb{D}$ be symmetric monoidal categories. We write $\mathbf{S M C}_{l}(\mathbb{D}, \mathbb{C})$ for the category of symmetric lax monoidal functors and monoidal natural transformations between them. The following pointwise extension of the tensor unit and tensor product on $\mathbb{C}$ extends to a symmetric monoidal structure on $\mathbf{S M C}_{l}(\mathbb{D}, \mathbb{C})$ :

$$
\dot{\mathbf{I}}(D)=\mathbf{I}, \quad(F \dot{\otimes} G)(D)=F D \otimes G D .
$$

(We note that the symmetry in $\mathbb{C}$ is used to make $F \dot{\otimes} G$ a symmetric lax monoidal functor.) Below by $[\mathbb{D}, \mathbb{C}]_{l}$ we mean the symmetric monoidal category $\left(\mathbf{S M C}_{l}(\mathbb{D}, \mathbb{C}), \dot{\mathbf{I}}, \dot{\otimes}\right)$ of symmetric lax monoidal functors and monoidal natural transformations between them.

\subsection{Graded Linear Exponential Comonad}

Fix a partially ordered semiring $(R, \leq, 0,+, 1, *)$. We introduce the main subject of this study, $R$-graded linear exponential comonad. This concept first appeared in [3, Definition 13] under the name exponential action. We adopt the following definition [7, Sect. 5.2], which is equivalent to the exponential action: 

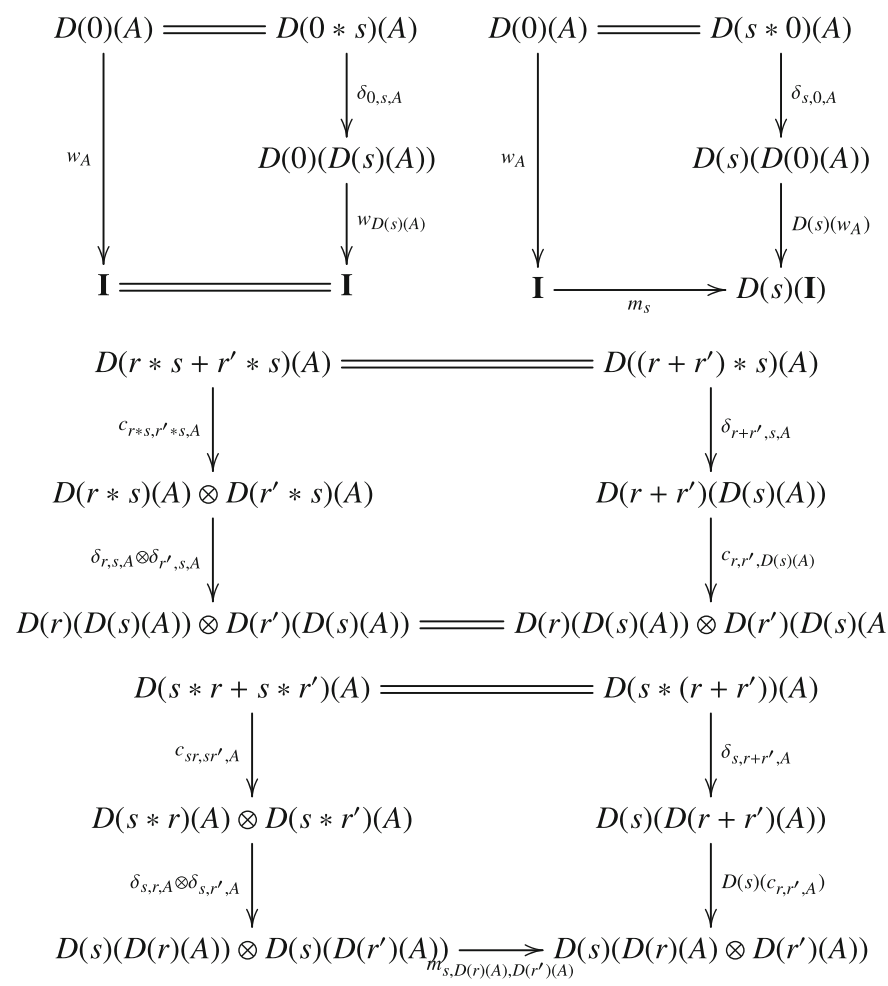

Fig. 1. Four equational axioms related to distributive law

Definition 1. An $R$-graded linear exponential comonad on a symmetric monoidal category $\mathbb{C}$ is a tuple $(D, w, c, \epsilon, \delta)$ where

- $D:(R, \leq) \rightarrow \mathbf{S M C}_{l}(\mathbb{C}, \mathbb{C})$ is a functor. Below we write $m_{r}: \mathbf{I} \rightarrow D(r)(\mathbf{I})$ and $m_{r, A, B}: D(r)(A) \otimes D(r)(B) \rightarrow D(r)(A \otimes B)$ for the symmetric lax monoidal structure of $D(r)$.

$-(D, w, c): R^{+} \rightarrow[\mathbb{C}, \mathbb{C}]_{l}$ is a symmetric colax monoidal functor.

$-(D, \epsilon, \delta): R^{*} \rightarrow\left(\mathbf{S M C}_{l}(\mathbb{C}, \mathbb{C}), \mathrm{Id}, \circ\right)$ is a colax monoidal functor.

They satisfy four equational axioms in Fig. 1. Moreover, we say that $D$ is an $R$ twist if $D r$ is strong monoidal for each $r \in R$, and $(D, \epsilon, \delta)$ is a strict monoidal functor (hence $D 1=\mathrm{Id}$ and $\left.D\left(r * r^{\prime}\right)=D r \circ D r^{\prime}\right)$.

When fully expanded, a graded linear exponential comonad specifies one functor $D:(R, \leq) \rightarrow[\mathbb{C}, \mathbb{C}]$ and 6 natural transformations:

$$
\begin{array}{ll}
m_{r}: D(r)(\mathbf{I}) \rightarrow \mathbf{I}, & m_{r, A, B}: D(r)(A \otimes B) \rightarrow D(r)(A) \otimes D(r)(B) \\
w_{A}: D(0)(A) \rightarrow \mathbf{I} & c_{r, r^{\prime}, A}: D\left(r+r^{\prime}\right)(A) \rightarrow D(r)(A) \otimes D\left(r^{\prime}\right)(A) \\
\epsilon_{A}: D(1)(A) \rightarrow A & \delta_{r, r^{\prime}, A}: D\left(r * r^{\prime}\right)(A) \rightarrow D(r)\left(D\left(r^{\prime}\right)(A)\right)
\end{array}
$$

satisfying more than 20 equational axioms. 
Example 1. Let $\mathbb{C}$ be a cartesian closed category. We take a partially ordered monoid $R^{\times}=(R, \leq, 1, \times)$ such that $(R, \leq)$ is a join semilattice and $\times$ preserves joins in both arguments. This condition makes the tuple $R=(R, \leq, \perp, \vee, 1, \times)$ a partially ordered semiring. We also take a lax monoidal functor $G: R^{\times} \rightarrow \mathbb{C}$. Then the functor $D:(R, \leq)^{o p} \rightarrow[\mathbb{C}, \mathbb{C}]$ defined by $\operatorname{Dr} A=G r \Rightarrow A$ extends to an $R^{o p}$-graded linear exponential comonad on $\mathbb{C}$ (here $R^{o p}$ is the orderopposite of $R$ ).

Example 2. Continuing the previous example, let $R=(D, \leq, \perp, \vee, \top, \wedge)$ be a distributive lattice, regarded as a partially ordered semiring. We consider the functor category $[D$, Set $]$, where $D$ is regarded as the discrete category of the carrier set $D$. We then define $G: R \rightarrow[D$, Set $]$ by $(G r) r^{\prime}=\emptyset$ if $r^{\prime} \not \leq r$, and $(G r) r^{\prime}=\{*\}$ if $r^{\prime} \leq r$. This $G$ extends to a lax monoidal functor of type $G$ : $R^{\times} \rightarrow[D$, Set $]$. From the construction in the previous example, $\operatorname{Dr} A=G r \Rightarrow A$ is a graded linear exponential comonad, which coincides with the masking functor given in [7, Theorem 2]. It behaves as $(D r A) r^{\prime}=\{*\}$ if $r^{\prime} \not \leq r$ and $(D r A) r^{\prime}=A r^{\prime}$ if $r^{\prime} \leq r$. This graded linear exponential comonad is used to model the level of information flow [7, Sect.6.1].

Example 3. Consider the category EPMet of extended pseudometric spaces ${ }^{1}$ and nonexpansive functions between them. It has a symmetric monoidal (closed) structure, whose unit is a terminal object, and whose tensor product is given by $(X, d) \otimes(Y, e)=(X \times Y, d+e)$. It also has the scaling modality $!_{r}(X, d)=(X, r d)$, where $r$ is an element of the ordered semiring of nonnegative extended reals, which we denote by $[0, \infty]$. The scaling modality is a $[0, \infty]$-twist with respect to the above symmetric monoidal structure.

The concept of $R$-graded linear exponential comonad is a generalization of non-graded linear exponential comonad [1, Definition 3]. This was first observed in $[3]$.

Theorem 1. A 1-graded linear exponential comonad on a symmetric monoidal category $\mathbb{C}$ is exactly a non-graded linear exponential comonad on $\mathbb{C}$.

On the other hand, 1-twists make monoidal structures cartesian:

Theorem 2. A 1-twist $D$ exists on a symmetric monoidal category $\mathbb{C}$ if and only if the symmetric monoidal structure of $\mathbb{C}$ is cartesian (i.e. I is terminal and $\otimes$ is a binary product).

Proof. If it exists, the functor part of $D$ must specify the identity functor $\operatorname{Id}_{\mathbb{C}}$ because of the strictness. Next, $(\mathrm{Id}, w, c)$ becomes a commutative monoid in $[\mathbb{C}, \mathbb{C}]_{l}$; especially $w, c$ are monoidal natural transformations. From $[17$, Corollary 17], the monoidal structure of $\mathbb{C}$ is cartesian. The converse construction is evident.

\footnotetext{
${ }^{1}$ Here, extended pseudometrics mean the pseudometrics that can return $+\infty$.
} 


\section{A Double-Category Theoretic Reformulation of Graded Linear Exponential Comonad}

Although it is in a reasonably compact form, the definition of graded linear exponential comonad is yet technical, and it indeed specifies a quite complex structure. The motivation of this study is to have a conceptually clean and compact definition of it.

Particularly, what is less clear in the definition is the extra four axioms related to the distributive law (Fig. 1). In the non-graded setting (i.e. when $R=1$ ), these four axioms reduces to simpler axioms, which can be viewed as the following conditions:

- comultiplication $\delta$ is a comonoid morphism, (item 4, Sect.7.4, [17]) and

- weakening $w$ and contraction $c$ are coalgebra morphisms (item 3, Sect. 7.4, [17]).

However, it is not obvious how to upgrade these axioms to the graded setting, because the concept of "graded coalgebra" and "graded comonoid" are not yet defined, at least for graded linear exponential comonads. Especially, the concept of graded coalgebra should be defined after the concept of graded linear exponential comonad, which we are going to define! From this circularity, the above view of the four axioms are not very helpful when upgrading them in the current situation.

It is therefore desirable to have an alternative account on four axioms in Fig. 1, which relies on a notion that already exists before graded linear exponential comonads. The key observation of this paper is that these four axioms are an instance of the axioms for 2-cells in the double category SMC of symmetric monoidal categories, introduced by Grandis and Paré [10, Sect. 2.3]. In SMC, a 2-cell consists of the following data:

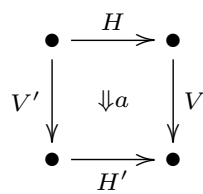

where each • is a (possibly distinct) symmetric monoidal category, horizontal morphisms $H, H^{\prime}$ are symmetric lax monoidal functors, vertical morphisms $V, V^{\prime}$ are symmetric colax monoidal functors, and $a: V \circ H \rightarrow H^{\prime} \circ V^{\prime}$ is a natural transformation (between underlying functors of $H, H^{\prime}, V, V^{\prime}$ ) making the following diagrams commute:
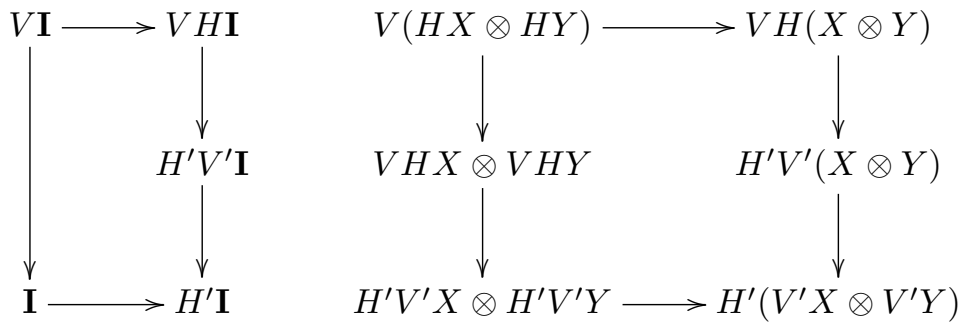
We note that when $V, V^{\prime}$ (resp. $H, H^{\prime}$ ) are identity functors, the above axioms are reduced to the ones for monoidal natural transformations of type $V \rightarrow V^{\prime}$ (resp. $H \rightarrow H^{\prime}$ ).

Let us see how 2-cell axioms (1) in SMC derives the four axioms in Fig. 1.

Proposition 1. In Definition 1, the four axioms (Fig. 1) can be replaced by the following statement: for each $r \in R$, both

$$
\delta_{r,-}: D(r *-) \rightarrow D r \circ D-, \quad \delta_{-, r}: D(-* r) \rightarrow D-\circ D r
$$

are 2-cells of the following type in $\mathbf{S M C}$ :
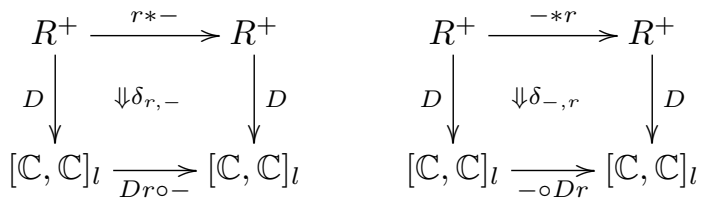

\section{Multicategory of Symmetric Lax Monoidal Multifunctors}

Proposition 1 says that by fixing one index of the doubly-indexed natural transformation $\delta_{-,=}: D(-*=) \rightarrow D-\circ D=$, we obtain a 2-cell in the double category SMC. However, $\delta$ itself does not live in SMC. In order to create a room to accommodate $\delta$ as a kind of 2-cell, we extend horizontal morphisms of SMC to multi-ary functors that are symmetric lax monoidal in each argument. We first study such multi-ary functors in this section.

Let $\mathbb{C}_{i}(1 \leq i \leq n)$ and $\mathbb{D}$ be symmetric monoidal categories. Intuitively, an $n$-ary functor $F: \mathbb{C}_{1} \times \cdots \times \mathbb{C}_{n} \rightarrow \mathbb{D}$ is symmetric lax monoidal in each argument if it comes with a structure making the functor $F\left(C_{1}, . .,-{ }_{m}, . ., C_{n}\right)$ : $\mathbb{C}_{m} \rightarrow \mathbb{D}$ symmetric lax monoidal for each $m \in\{1, \cdots, n\}$ and $C_{i} \in \mathbb{C}_{i}, i \in$ $\{1, \cdots, n\} \backslash\{m\}$. Moreover, these symmetric lax monoidal structures commute with each other in a coherent manner.

To formally define such multi-ary symmetric lax monoidal functors, we introduce a notation for sequences. For a sequence $C=C_{1}, \cdots, C_{n}$ of mathematical objects, a natural number $1 \leq i \leq n$ and another sequence $D$, by $C[i: D]$ we mean the sequence obtained by replacing $C_{i}$ with $D$. For instance, $(1,3,5)[2: X, Y]=1, X, Y, 5$. When $D$ is empty, $C[i:]$ stands for the sequence obtained by removing the $i$-th element of $C$.

Definition 2. A symmetric lax monoidal multifunctor of type $\left(\mathbb{C}_{1}, \cdots, \mathbb{C}_{n}\right) \rightarrow$ $\mathbb{D}$ consists of a functor and a family of natural transformations indexed by $1 \leq$ $i \leq n$ :

$$
\begin{aligned}
& F: \mathbb{C}_{1} \times \cdots \times \mathbb{C}_{n} \rightarrow \mathbb{D} \\
& \phi_{C[i:]}^{i}: \mathbf{I} \rightarrow F(C[i: \mathbf{I}]) \quad\left(C \in \mathbb{C}_{1} \times \cdots \times \mathbb{C}_{n}\right) \\
& \phi_{C[i: X, Y]}^{i}: F(C[i: X]) \otimes F(C[i: Y]) \rightarrow F(C[i: X \otimes Y]) \quad\left(C \in \mathbb{C}_{1} \times \cdots \times \mathbb{C}_{n}, X, Y \in \mathbb{C}_{i}\right)
\end{aligned}
$$


such that:

1. For each $C \in \mathbb{C}_{1} \times \cdots \times \mathbb{C}_{n}$ and $1 \leq i \leq n$, The tuple $(F(C[i:-])$, $\left.\phi_{C[i:]}^{i}, \phi_{C[i:-,=]}^{i}\right)$ is a symmetric lax monoidal functor from $\mathbb{C}_{i}$ to $\mathbb{D}$. We denote it by $F(C / i)$.

2. The following equalities hold for each $C \in \mathbb{C}_{1} \times \cdots \times \mathbb{C}_{n}$ and $1 \leq i<j \leq n$ :

$-\phi_{C[j: \mathbf{I}][i:]}^{i}=\phi_{C[i: \mathbf{I}][j:]}^{j}$

$-\phi_{C[i: \mathbf{I}][j: P, Q]}^{j} \circ\left(\phi_{C[j: P][i:]}^{i} \otimes \phi_{C[j: Q][i:]}^{i}\right)=\phi_{C[j: P \otimes Q][i:]}^{i} \circ \iota$

$-\phi_{C[j: \mathbf{I}][i: P, Q]}^{i} \circ\left(\phi_{C[i: P][j:]}^{j} \otimes \phi_{C[i: Q][j:]}^{j}\right)=\phi_{C[i: P \otimes Q][j:]}^{j} \circ \iota$

$-\phi_{C[i: X \otimes Y][j: P, Q]}^{j} \circ\left(\phi_{C[j: P][i: X, Y]}^{i} \otimes \phi_{C[j: Q][i: X, Y]}^{i}\right)=\phi_{C[j: P \otimes Q][i: X, Y]}^{i} \circ$ $\left(\phi_{C[i: X][j: P, Q]}^{j} \otimes \phi_{C[i: Y][j: P, Q]}^{j}\right) \circ \tau$.

We note that a symmetric lax monoidal multifunctor of type ()$\rightarrow \mathbb{D}$ is just an object in $\mathbb{D}$, because all natural transformations vanish and only the functor of type $1 \rightarrow \mathbb{D}$ remains.

Example 4. Let us see how the definition of a binary symmetric lax monoidal multifunctor $M:(\mathbb{C}, \mathbb{C}) \rightarrow \mathbb{C}$ is unfolded. It consists of a functor $M: \mathbb{C} \times \mathbb{C} \rightarrow \mathbb{C}$ and the following natural transformations:

$$
\begin{aligned}
& \phi_{C}^{1}: \mathbf{I} \rightarrow M(\mathbf{I}, C), \quad \phi_{X, Y, C}^{1}: M(X, C) \otimes M(Y, C) \rightarrow M(X \otimes Y, C) \\
& \phi_{C}^{2}: \mathbf{I} \rightarrow M(C, \mathbf{I}), \quad \phi_{C, X, Y}^{2}: M(C, X) \otimes M(C, Y) \rightarrow M(C, X \otimes Y)
\end{aligned}
$$

such that

1. For each $C \in \mathbb{C},\left(M(-, C), \phi_{C}^{1}, \phi_{-,=, C}^{1}\right)$ and $\left(M(C,-), \phi_{C}^{2}, \phi_{C,-,=}^{2}\right)$ are symmetric lax monoidal functors of type $\mathbb{C} \rightarrow \mathbb{C}$.

2. The following coherence axioms holds:

$$
\begin{aligned}
& \phi_{\mathbf{I}}^{1}=\phi_{\mathbf{I}}^{2}, \quad \phi_{C \otimes C^{\prime}}^{1} \circ \iota=\phi_{\mathbf{I}, C, C^{\prime}}^{2} \circ\left(\phi_{C}^{1} \otimes \phi_{C^{\prime}}^{1}\right), \quad \phi_{C \otimes C^{\prime}}^{2} \circ \iota=\phi_{C, C^{\prime}, \mathbf{I}}^{1} \circ\left(\phi_{C}^{2} \otimes \phi_{C^{\prime}}^{2}\right) \\
& \phi_{C \otimes C^{\prime}, D, D^{\prime}}^{2} \circ\left(\phi_{C, C^{\prime}, D}^{1} \otimes \phi_{C, C^{\prime}, D^{\prime}}^{1}\right)=\phi_{C, C^{\prime}, D \otimes D^{\prime}}^{1} \circ\left(\phi_{C, D, D^{\prime}}^{2} \otimes \phi_{C^{\prime}, D, D^{\prime}}^{2}\right) \circ \tau
\end{aligned}
$$

We will later use the following binary symmetric lax monoidal multifunctors. Let $R$ be a partially ordered semiring and $\mathbb{C}$ be a symmetric monoidal category.

1. The multiplication $(*)$ is a symmetric lax monoidal multifunctor of type $\left(R^{+}, R^{+}\right) \rightarrow R^{+}$.

2. The evaluation functor $e v:[\mathbb{C}, \mathbb{C}]_{l} \times \mathbb{C} \rightarrow \mathbb{C}$ extends to a symmetric lax monoidal multifunctor of type $\left([\mathbb{C}, \mathbb{C}]_{l}, \mathbb{C}\right) \rightarrow \mathbb{C}$.

3. The functor composition (o) extends to a symmetric lax monoidal multifunctor of type $\left([\mathbb{C}, \mathbb{C}]_{l},[\mathbb{C}, \mathbb{C}]_{l}\right) \rightarrow[\mathbb{C}, \mathbb{C}]_{l}$.

Note that $(*)$ is symmetric strict monoidal in each argument, while (o), ev are symmetric strict monoidal in the first argument, and symmetric lax monoidal in the second argument. 
Next, for symmetric lax monoidal multifunctors $(F, \phi):\left(\mathbb{C}_{1}, \cdots, \mathbb{C}_{n}\right) \rightarrow \mathbb{D}$ and $\left(G_{i}, \gamma(i)\right):\left(\mathbb{B}_{i, 1}, \cdots, \mathbb{B}_{i, m_{i}}\right) \rightarrow \mathbb{C}_{i}(1 \leq i \leq n)$, we define their multicomposition. First, we define a bijection $(/):\left\{(i, j) \mid 1 \leq i \leq n, 1 \leq j \leq m_{i}\right\} \rightarrow$ $\left\{1, \cdots, \sum_{1 \leq i \leq n} m_{i}\right\}$, and represent a number in the latter set as the pair of numbers uniquely determined by $(/)$ in the former set. Then the multicomposition is given by the following $(H, \eta)$ :

$$
\begin{aligned}
H & =F \circ\left(G_{1} \times \cdots \times G_{n}\right) \\
\eta_{\left(B_{1}, \cdots, B_{n}\right)[i / j:]}^{i / j} & =F\left(\left(G B_{1}, \cdots, G B_{n}\right)\left[i: \gamma(i)_{B_{i}[j:]}^{j}\right] \circ \phi_{\left(G B_{1}, \cdots, G B_{n}\right)[i:]}^{i}\right. \\
\eta_{\left(B_{1}, \cdots, B_{n}\right)[i / j: X, Y]}^{i / j} & =F\left(\left(G B_{1}, \cdots, G B_{n}\right)\left[i: \gamma(i)_{B_{i}[j: X, Y]}^{j}\right] \circ \phi_{\left(G B_{1}, \cdots, G B_{n}\right)\left[i: G\left(B_{i}[j: X]\right), G\left(B_{i}[j: Y]\right)\right]}^{i}\right.
\end{aligned}
$$

Theorem 3. Symmetric monoidal categories, symmetric lax monoidal multifunctors, and the above multi-composition form a multicategory $\mathbf{M S M C}$.

Proof (Proof sketch). To check that symmetric lax monoidal multifunctors are closed under multicomposition, the key case is when $n=2, m_{1}=m_{2}=1$ and $n=1, m_{1}=2$.

In $\mathbf{M S M C}$ we consider monoids and monoid actions. A monoid is a tuple $(\mathbb{C}, U:() \rightarrow \mathbb{C}, M:(\mathbb{C}, \mathbb{C}) \rightarrow \mathbb{C})$ of a symmetric monoidal category $\mathbb{C}$ and symmetric lax monoidal multifunctors $U, M$ such that

$$
\mathrm{Id}=M \circ(\mathrm{Id}, U), \quad \mathrm{Id}=M \circ(U, \mathrm{Id}), \quad M \circ(\mathrm{Id}, M)=M \circ(M, \mathrm{Id}) .
$$

An action of a monoid $(\mathbb{C}, U, M)$ on a symmetric monoidal category $\mathbb{D}$ is a symmetric lax monoidal multifunctor $A:(\mathbb{C}, \mathbb{D}) \rightarrow \mathbb{D}$ such that

$$
A \circ(U, \mathrm{Id})=\mathrm{Id}, \quad A \circ(\mathrm{Id}, A)=A \circ(M, \mathrm{Id}) .
$$

By unfolding the definition, a monoid $(\mathbb{C}, U, M)$ in $\mathbf{M S M C} \mathbf{C}_{l}$ equips $\mathbb{C}$ with an additional strict monoidal structure $(U, M)$. The argument-wise symmetric lax monoidal structure on $M$ becomes a lax distributivity (see Example 4). Thus we call a monoid in $\mathbf{M S M C} \mathbf{M}_{l}$ a lax distributive strict rig category. It has a smaller set of coherence axioms than the one given by Laplaza in [15], thanks to the strictness of $(U, M)$.

Example 5 (Continued from Example 4$).\left(R^{+}, 1, *\right)$ and $\left([\mathbb{C}, \mathbb{C}]_{l}\right.$, Id, o) are both lax distributive strict rig categories. Both monoids acts on themselves. The latter monoid acts on $\mathbb{C}$ with the evaluation functor $e v$.

\section{Graded Linear Exponential Comonads as Vertical Monoid Homomorphisms}

We now extend the double category SMC of Grandis and Paré by replacing horizontal morphisms with symmetric lax monoidal multifunctors. The concept of 2-cells in SMC is also replaced by prisms - the reason of the name is because they are placed in the middle of the space surrounded by two horizontal multifunctors and vertical morphisms. Such a prism is defined to be a natural transformation that is a 2-cell of SMC in each argument. 
Definition 3. Let $F:\left(\mathbb{C}_{1}, \cdots, \mathbb{C}_{n}\right) \rightarrow \mathbb{D}$ and $G:\left(\mathbb{E}_{1}, \cdots, \mathbb{E}_{n}\right) \rightarrow \mathbb{F}$ be symmetric lax monoidal multifunctors and $V_{i}: \mathbb{C}_{i} \rightarrow \mathbb{E}_{i}(1 \leq i \leq n)$ and $W: \mathbb{D} \rightarrow \mathbb{F}$ be symmetric colax monoidal functors. A prism $\alpha$ of type $\left(V_{1}, \cdots, V_{n}\right) \rightarrow W: F \rightarrow$ $G$, which is depicted as

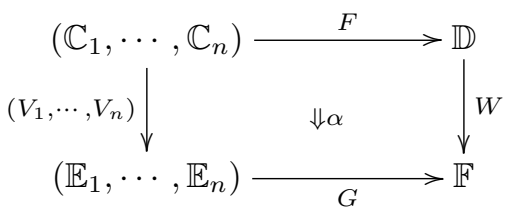

is a natural transformation $\alpha: W \circ F \rightarrow G \circ\left(V_{1} \times \cdots \times V_{n}\right)$ such that for each $C \in \prod_{i=1}^{n} \mathbb{C}_{i}$ and $1 \leq i \leq n, \alpha_{C[i:-]}$ is a 2-cell of the following type in the double category SMC:

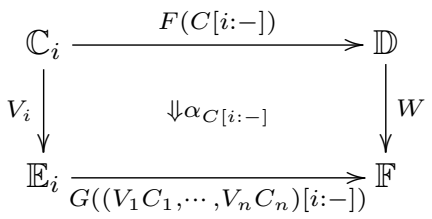

We note that when $n=0$, a prism $\alpha:() \rightarrow W: F \rightarrow G$ is simply a morphism $\alpha: W F \rightarrow G$ in $\mathbb{F}$.

Proposition 2. Let $D: R^{+} \rightarrow[\mathbb{C}, \mathbb{C}]_{l}$ be a symmetric colax monoidal functor and $\delta$ be a prism of type $(D, D) \rightarrow D:(*) \rightarrow(\circ)$, where $(*)$ and $(\circ)$ are symmetric lax monoidal multifunctors appeared in Example 4. Then for each $r \in R, \delta_{r,-}$ and $\delta_{-, r}$ are 2-cells of the following type in SMC:
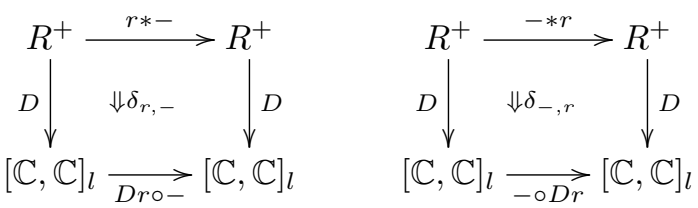

Like double categories, composition of prisms can be done in two directions. Consider the following prisms $(1 \leq i \leq n)$.
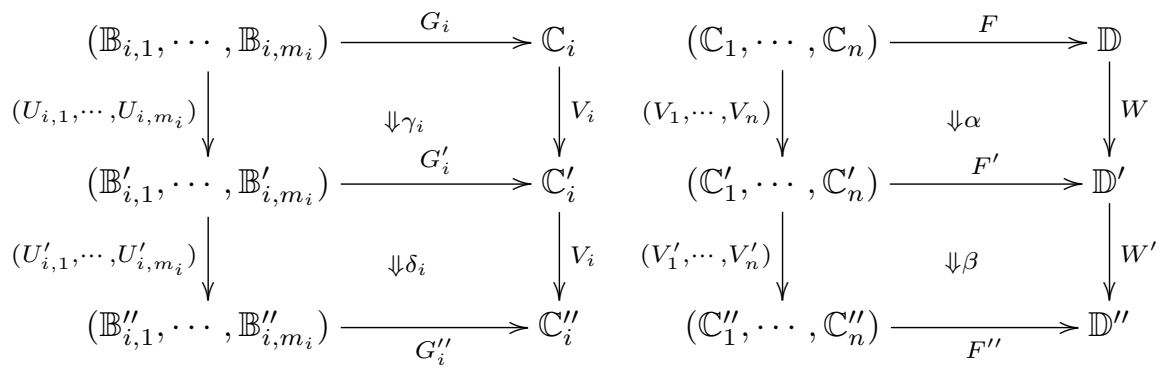
Then define vertical composition and horizontal multicomposition of prisms by the following (ordinary) natural transformations:

$$
\begin{aligned}
\beta \odot \alpha & =\left(\beta \circ\left(V_{1} \times \cdots \times V_{n}\right)\right) \bullet\left(W^{\prime} \circ \alpha\right) \\
\alpha \circledast\left(\gamma_{1}, \cdots, \gamma_{n}\right) & =\left(F^{\prime} \circ\left(\gamma_{1} \times \cdots \times \gamma_{n}\right)\right) \bullet\left(\alpha \circ\left(G_{1} \times \cdots \times G_{n}\right)\right)
\end{aligned}
$$

where $\bullet$ on the right hand side is the vertical composition of natural transformations.

Proposition 3. In the above setting,

1. $\beta \odot \alpha$ is a prism of type $\left(V_{1}^{\prime} \circ V_{1}, \cdots, V_{n}^{\prime} \circ V_{n}\right) \rightarrow W^{\prime} \circ W: F \rightarrow F^{\prime \prime}$.

2. $\alpha \circledast\left(\gamma_{1}, \cdots, \gamma_{n}\right)$ is a prism of type $\left(U_{1,1}, \cdots, U_{n, m_{n}}\right) \rightarrow W$ : $F \circ$ $\left(G_{1}, \cdots, G_{n}\right) \rightarrow F^{\prime} \circ\left(G_{1}^{\prime}, \cdots, G_{n}^{\prime}\right)$.

3. The interchange law holds:

$$
\left(\beta \circledast\left(\delta_{1}, \cdots, \delta_{n}\right)\right) \odot\left(\alpha \circledast\left(\gamma_{1}, \cdots, \gamma_{n}\right)\right)=(\beta \odot \alpha) \circledast\left(\delta_{1} \odot \gamma_{1}, \cdots, \delta_{n} \odot \gamma_{n}\right) .
$$

Definition 4. Let $(\mathbb{C}, U, M),\left(\mathbb{D}, U^{\prime}, M^{\prime}\right)$ be monoids in $\mathbf{M S M C}$. A vertical monoid homomorphism consists of a symmetric colax monoidal functor $A: \mathbb{C} \rightarrow$ $\mathbb{D}$ and prisms $\epsilon:() \rightarrow A: U \rightarrow U^{\prime}$ and $\delta:(A, A) \rightarrow A: M \rightarrow M^{\prime}:$

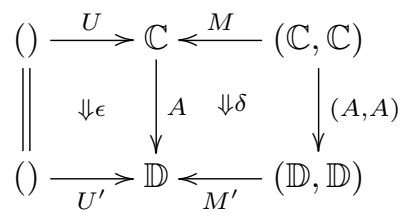

such that the following prism equalities hold:

$$
\delta \circledast(\mathrm{id}, \epsilon)=\mathrm{id}, \quad \delta \circledast(\epsilon, \mathrm{id})=\mathrm{id}, \quad \delta \circledast(\mathrm{id}, \delta)=\delta \circledast(\delta, \mathrm{id}) .
$$

The above prism equalities amounts to the following equality of natural transformations:

$$
\begin{aligned}
& M^{\prime}(A X, \epsilon) \circ \delta_{X, U}=\mathrm{id} \quad M^{\prime}(\epsilon, A X) \circ \delta_{U, X}=\mathrm{id} \\
& M^{\prime}\left(A X, \delta_{Y, Z}\right) \circ \delta_{X, M(Y, Z)}=M^{\prime}\left(\delta_{X, Y}, A Z\right) \circ \delta_{M(X, Y), Z}
\end{aligned}
$$

With this concept, we can concisely capture $R$-graded linear exponential comonads:

Theorem 4. There is a bijective correspondence between

1. A vertical monoid homomorphism $(D, \epsilon, \delta)$ from $\left(R^{+}, 1, *\right)$ to $\left([\mathbb{C}, \mathbb{C}]_{l}, \mathrm{Id}, \circ\right)$.

2. An $R$-graded linear exponential comonad on $\mathbb{C}$.

Vertical monoid homomorphisms vertically compose. Therefore we can extend a graded linear exponential comonad (as a vertical monoid homomorphism) by stacking vertical monoid homomorphisms. 
Proposition 4. Let $R, S$ be partially ordered semirings. Then a vertical monoid homomorphism from $\left(R^{+}, 1_{R}, *_{R}\right)$ to $\left(S^{+}, 1_{S}, *_{S}\right)$ bijectively corresponds to a monotone function $h:\left(R, \leq_{R}\right) \rightarrow\left(S, \leq_{S}\right)$ such that $h\left(\sum_{R} r_{i}\right) \leq \sum_{S} h\left(r_{i}\right)$ and $h\left(\prod_{R} r_{i}\right) \leq \prod_{S} h\left(r_{i}\right)$ (which we call colax homomorphism).

Proposition 5. Let $F \dashv U: \mathbb{C} \rightarrow \mathbb{D}$ be a symmetric lax monoidal adjunction. Then the functor $V^{F \dashv U}$ defined by $V^{F \dashv U} H=F \circ H \circ U$ is a vertical monoid homomorphism from $\left([\mathbb{C}, \mathbb{C}]_{l}, \mathrm{Id}, \circ\right)$ to $\left([\mathbb{D}, \mathbb{D}]_{l}, \mathrm{Id}, \circ\right)$.

Proof. Let $F \dashv U: \mathbb{C} \rightarrow \mathbb{D}$ be a symmetric lax monoidal adjunction. From Kelly's doctrinal adjunction, $F$ is symmetric strong monoidal, hence so is $F \circ-$ in the following diagram:

$$
V^{F \dashv U}=[\mathbb{C}, \mathbb{C}]_{l} \stackrel{F \circ-}{\longrightarrow}[\mathbb{C}, \mathbb{D}]_{l} \stackrel{-\circ U}{\longrightarrow}[\mathbb{D}, \mathbb{D}]_{l}
$$

Next, $-\circ U$ above is always symmetric strict monoidal. By composing them, we obtain that $V^{F \dashv U}$ is symmetric strong, hence colax monoidal. We next introduce prisms $(\epsilon, \delta)$ of the following type:

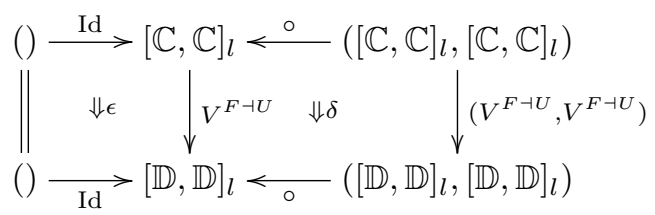

We define $\epsilon$ to be the counit of the adjunction $F \dashv U$, which is monoidal natural, and $\delta$ be the following natural transformation:

$$
\delta_{H_{1}, H_{2}}=V^{F \dashv U}\left(H_{1} \circ \eta \circ H_{2}\right): V^{F \dashv U}\left(H_{1} \circ H_{2}\right) \rightarrow V^{F \dashv U} H_{1} \circ V^{F \dashv U} H_{2}
$$

It is routine to check that this satisfies the axioms of prism.

Theorem 5. Let $R$ be a partially ordered semiring and $D$ be an $R$-graded linear exponential comonad on a symmetric monoidal category $\mathbb{C}$. We moreover let $S$ be another partially ordered semiring, $h: S \rightarrow R$ be a colax homomorphism and $F \dashv U: \mathbb{C} \rightarrow \mathbb{D}$ be a symmetric lax monoidal adjunction. Then the following composite of vertical monoid homomorphisms is an S-graded linear exponential comonad on $\mathbb{D}$.

$$
\left(S^{+}, 1_{S}, *_{S}\right) \stackrel{h}{\longrightarrow}\left(R^{+}, 1_{R}, *_{R}\right) \stackrel{D}{\longrightarrow}\left([\mathbb{C}, \mathbb{C}]_{l}, \operatorname{Id}_{\mathbb{C}}, \circ\right) \stackrel{V^{F \dashv U}}{\longrightarrow}\left([\mathbb{D}, \mathbb{D}]_{l}, \operatorname{Id}_{\mathbb{D}}, \circ\right)
$$

We call the above composite the extension of $D$ with $F \dashv U$ and $h$.

\section{From Monoid Actions to Graded Comonoid-Coalgebras}

Let $(D, \epsilon, \delta):\left(R^{+}, 1, *\right) \rightarrow\left([\mathbb{C}, \mathbb{C}]_{l}, \mathrm{Id}, \circ\right)$ be an $R$-graded linear exponential comonad as a vertical monoid homomorphism. The prism equations in 
Definition 4 suggests that the vertical monoid homomorphism itself can be seen as a monoid. We can thus consider monoid actions of $(D, \epsilon, \delta)$ : it consists of a prism

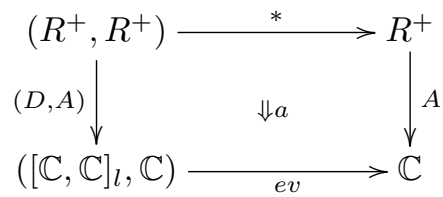

such that the following prism equations hold:

$$
a \circledast(\delta, \mathrm{id})=a \circledast(\mathrm{id}, a), \quad a \circledast(\epsilon, \mathrm{id})=\mathrm{id} .
$$

We note that this makes sense because $(*)$ and $e v$ are also monoid actions in $\mathbf{M S M C}_{l}$; see Example 5. By unfolding this definition, we obtain the following structure, which we name graded comonoid-coalgebra.

Definition 5. Let $R$ be a partially ordered semiring. An $R$-graded comonoidcoalgebra of an $R$-graded linear exponential comonad $(D, w, c, \epsilon, \delta)$ on a symmetric monoidal category $\mathbb{C}$ is a tuple $(A, a, u, o)$ such that

- $(A, u, o): R^{+} \rightarrow \mathbb{C}$ is a symmetric colax monoidal functor.

- $a_{r, r^{\prime}}: A\left(r * r^{\prime}\right) \rightarrow D(r)\left(A\left(r^{\prime}\right)\right)$ is a natural transformation.

They satisfy the following six equational axioms:

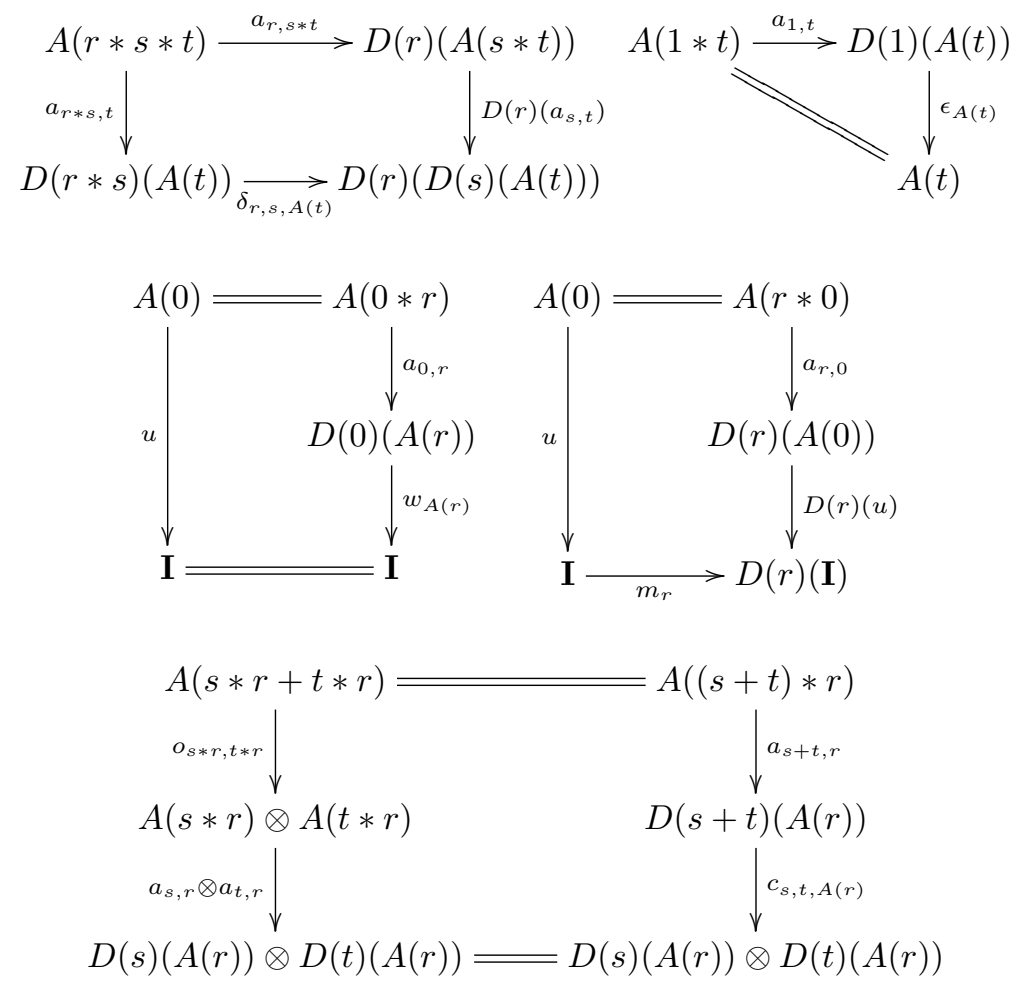




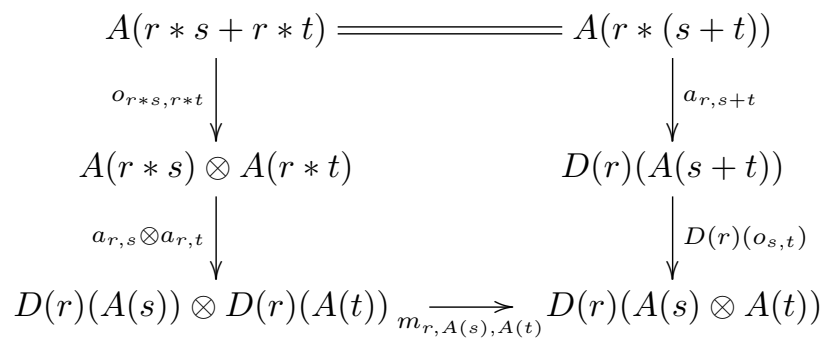

$A$ morphism from an $R$-graded comonoid-coalgebra $(A, a, u, o)$ to another $(B, b, v, p)$ is a monoidal natural transformation $h:(A, u, o) \rightarrow(B, v, p)$ such that $h$ satisfies:

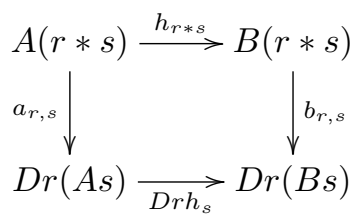

We write $C(\mathbb{C}, D)$ for the category of $R$-graded comonoid-coalgebras of $D$.

Proposition 6. Let $R$ be a partially ordered semiring and $(D, w, c, \epsilon, \delta)$ be an $R$-graded linear exponential comonad on a symmetric monoidal category $\mathbb{C}$. The following gives a symmetric monoidal structure on $C(\mathbb{C}, D)$ :

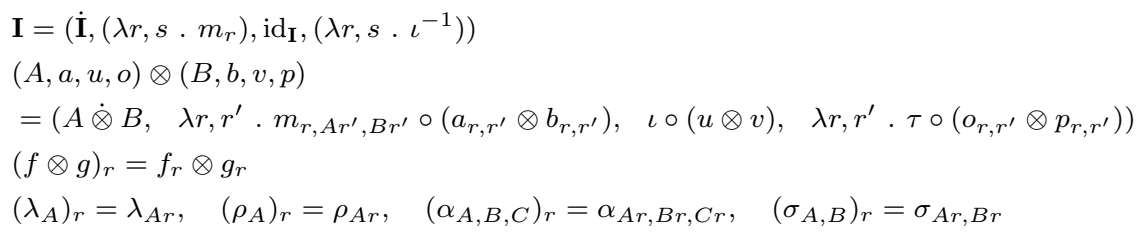

When $R=1$, The category $C(\mathbb{C}, D)$ reduces to the category of EilenbergMoore coalgebras of the non-graded linear exponential comonad.

Theorem 6. Let $(D, w, c, \epsilon, \delta)$ be a 1-graded linear exponential comonad on a symmetric monoidal category $\mathbb{C}$. Then the category $C(\mathbb{C}, D)$ is strong monoidally isomorphic to the category $\mathbb{C}^{D}$ of Eilenberg-Moore coalgebras of the comonad $(D, \epsilon, \delta)$.

Like $\mathbb{C}^{D}$, there is a symmetric lax monoidal adjunction of the following type:

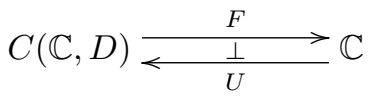

but this itself is not enough to recover $D-D$ takes two arguments, while the composite $F \circ U$ is only equal to the symmetric lax monoidal comonad $D 1$ on $\mathbb{C}$. 
The category $C(\mathbb{C}, D)$ actually carries an $R$-twist $T$, which acts on comonoidcoalgebras as follows:

$$
\operatorname{Tr}(A, \cdots)=(A(-* r), \cdots)
$$

and $D$ is recovered as the extension of $T$ with the adjunction $F \dashv U$ (Theorem 5 ).

Theorem 7. Let $R$ be a partially ordered semiring and $(D, w, c, \epsilon, \delta)$ be an $R$ graded linear exponential comonad on a symmetric monoidal category $\mathbb{C}$.

1. The functor $F: C(\mathbb{C}, D) \rightarrow \mathbb{C}$ given by $F(A, a, u, o)=A 1$ and $F h=h_{1}$ is symmetric strict monoidal, and has a symmetric lax monoidal right adjoint $U$ : $\mathbb{C} \rightarrow C(\mathbb{C}, D)$, whose object part is given by $U A=\left(\lambda r . \operatorname{Dr} A, \lambda r, r^{\prime} . \delta_{r, r^{\prime}, A}, w_{A}\right.$, $\left.\lambda r, r^{\prime} . c_{r, r^{\prime}, A}\right)$.

2. The following data give an $R$-twist $T$ on $C(\mathbb{C}, D)$ :

$$
\begin{aligned}
& \operatorname{Tr} A=\left(\lambda s . A(s * r), \quad \lambda s, s^{\prime} . a_{s, s^{\prime} * r}, \quad u, \quad \lambda s, s^{\prime} . o_{s * r, s^{\prime} * r}\right), \quad(T r h)_{t}=h_{t * r} \\
& \left(m_{r}^{T}\right)_{t}=\operatorname{id}_{\mathbf{I}}, \quad\left(m_{r, A, B}^{T}\right)_{t}=\operatorname{id}_{A(t * r) \otimes B(t * r)}, \quad\left(w_{A}^{T}\right)_{t}=u, \quad\left(c_{r, s, A}^{T}\right)_{t}=o_{t * r, t * s} .
\end{aligned}
$$

Here, $A=(A, a, u, o)$ and $B$ are $R$-graded comonoid coalgebras. From the definition of twists, $\epsilon^{T}, \delta^{T}$ are identities.

3. The extension of $D$ with $F \dashv U$ (Theorem 5) coincides with the $R$-graded linear exponential comonad D.

The following classic result [1, Theorem 6-1] can be reproved by Theorem 7 .

Corollary 1. Let $\mathbb{C}$ be a symmetric monoidal category and Let $D$ be a nongraded linear exponential comonad on $\mathbb{C}$. The canonical symmetric monoidal structure on the category $\mathbb{C}^{D}$ of Eilenberg-Moore coalgebras of $D$ is cartesian.

Proof. From Theorem $1, D$ is a 1 -graded linear exponential comonad on $\mathbb{C}$. Therefore $C(\mathbb{C}, D)$ has a 1 -twist by Theorem 7-3. Therefore the symmetric monoidal structure of $C(\mathbb{C}, D)$ is cartesian by Theorem 2. Finally, $C(\mathbb{C}, D)$ is strong monoidally isomorphic to $\mathbb{C}^{D}$ by Theorem 6 , hence the symmetric monoidal structure of $\mathbb{C}^{D}$ is also cartesian.

We show the finality of the category of graded comonoid-coalgebras. Let $R$ be a partially ordered semiring and $D$ be an $R$-graded linear exponential comonad on a symmetric monoidal category $\mathbb{C}$. We define a resolution of $D$ to be a pair of a symmetric lax monoidal adjunction $J \dashv K: \mathbb{E} \rightarrow \mathbb{C}$ and an $R$-twist $\left(S, w^{S}, c^{S}\right)$ on $\mathbb{E}$ such that the extension of $S$ with $J \dashv K$ is equal to $D$. Then the following set of data becomes a strong monoidal functor $\left(M, m^{M}, m_{E, E^{\prime}}^{M}\right): \mathbb{E} \rightarrow C(\mathbb{C}, D)$ :

$$
\begin{aligned}
& M E=\left(\begin{array}{lllll}
\lambda r . J(S r) E, & \left.\lambda r, r^{\prime} . J(S r) \eta_{S r^{\prime} E}^{J \dashv K}, \quad\left(m^{J}\right)^{-1} \circ w_{E}^{S}, \quad \lambda r, r^{\prime} .\left(m_{S r E, S r^{\prime} E}^{J}\right)^{-1} \circ J c_{r, r^{\prime}, E}^{S}\right)
\end{array}\right. \\
& (M f)_{r}=J(S r) f, \quad\left(m^{M}\right)_{r}=J\left(m_{r}^{S}\right) \circ m^{J}, \quad\left(m_{E, E^{\prime}}^{M}\right)_{r}=J\left(m_{r, E, E^{\prime}}^{S}\right) \circ m_{S r E, S r E^{\prime}}^{J}
\end{aligned}
$$

(recall that $S r, J$ are both symmetric strong monoidal). 
Theorem 8. The above $M$ is the unique symmetric strong monoidal functor such that:

1. Equality of symmetric lax monoidal functors $M \circ K=U$ and $F \circ M=J$ hold.

2. Let $M^{*}=-\circ M$ and $M_{*}=M \circ-$ be induced symmetric strict (resp. strong) monoidal functors. Then the following square of symmetric colax monoidal functors commutes.

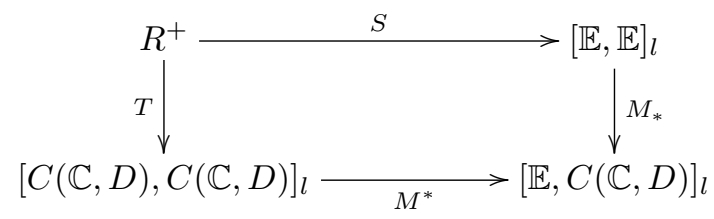

\section{Conclusion}

We have given a concise characterization of graded linear exponential comonad as a vertical monoid homomorphism $(D, \epsilon, \delta)$ from $\left(R^{+}, 1, *\right)$ to $\left([\mathbb{C}, \mathbb{C}]_{l}, \mathrm{Id}, \circ\right)$. This characterization is built upon a combination of the theory of symmetric lax monoidal multifunctors and Grandis and Paré's double category of symmetric monoidal categories. After this characterization, we considered monoid actions, and derived the concept of graded comonoid-coalgebras. The category of graded comonoid-coalgebras are shown to give a resolution of the graded linear exponential comonad $D$. These results are consistent with the theory of non-graded linear exponential comonads developed in [1].

It remains to be seen if the category of graded comonoid-coalgebras can be constructed in a purely double-category theoretic way. In non-graded case, there are other type of categorical models of exponential modality using Lafont category and Seely category [17]. Graded version of these categories are also an interesting research topic.

Acknowledgment. The author is grateful to Marco Gaboardi, Naohiko Hoshino, Flavien Breuvart, Soichiro Fujii and Paul-Andrè Melliès for many fruitful discussions. This research was supported by JSPS KAKENHI Grant Number JP15K00014 and ERATO Hasuo Metamathematics for Systems Design Project (No. JPMJER1603), JST.

\section{References}

1. Benton, N., Bierman, G., de Paiva, V., Hyland, M.: Linear $\lambda$-calculus and categorical models revisited. In: Börger, E., Jäger, G., Kleine Büning, H., Martini, S., Richter, M.M. (eds.) CSL 1992. LNCS, vol. 702, pp. 61-84. Springer, Heidelberg (1993). https://doi.org/10.1007/3-540-56992-8_6

2. Breuvart, F., Pagani, M.: Modelling coeffects in the relational semantics of linear logic. In: Kreutzer, S. (ed.) 24th EACSL Annual Conference on Computer Science Logic, CSL 2015, 7-10 September 2015, Berlin, Germany, vol. 41. LIPIcs, pp. 567581. Schloss Dagstuhl - Leibniz-Zentrum fuer Informatik (2015) 
3. Brunel, A., Gaboardi, M., Mazza, D., Zdancewic, S.: A core quantitative coeffect calculus. In: Shao [24], pp. 351-370

4. Dal Lago, U., Schöpp, U.: Functional programming in sublinear space. In: Gordon, A.D. (ed.) ESOP 2010. LNCS, vol. 6012, pp. 205-225. Springer, Heidelberg (2010). https://doi.org/10.1007/978-3-642-11957-6_12

5. de Amorim, A.A., Gaboardi, M., Hsu, J., Katsumata, S., Cherigui, I.: A semantic account of metric preservation. In: Castagna, G., Gordon, A.D. (eds.) Proceedings of the 44th ACM SIGPLAN Symposium on Principles of Programming Languages, POPL 2017, Paris, France, 18-20 January 2017, pp. 545-556. ACM (2017)

6. Durov, N.: New approach to Arakelov geometry. arXiv: 0704.2030 v1 [math AG], April 2008

7. Gaboardi, M., Katsumata, S.-Y., Orchard, D.A., Breuvart, F., Uustalu, T.: Combining effects and coeffects via grading. In: Garrigue, J., Keller, G., Sumii, E. (eds.) Proceedings of the 21st ACM SIGPLAN International Conference on Functional Programming, ICFP 2016, Nara, Japan, 18-22 September 2016, pp. 476-489. ACM (2016)

8. Ghica, D.R., Smith, A.I.: Bounded linear types in a resource semiring. In: Shao [24], pp. 331-350

9. Girard, J.-Y., Scedrov, A., Scott, P.J.: Bounded linear logic: a modular approach to polynomial-time computability. Theoret. Comput. Sci. 97(1), 1-66 (1992)

10. Grandis, M., Paré, R.: Adjoint for double categories. Cahiers de Topologie et Gomtrie Diffrentielle Catgoriques 45(3), 193-240 (2004)

11. Hyland, M., Power, J.: Pseudo-commutative monads and pseudo-closed 2categories. J. Pure Appl. Algebra 175(1), 141-185 (2002). Special Volume celebrating the 70th birthday of Professor Max Kelly

12. Jacobs, B., Löding, C. (eds.): FoSSaCS 2016. LNCS, vol. 9634. Springer, Heidelberg (2016). https://doi.org/10.1007/978-3-662-49630-5

13. Katsumata, S.: Parametric effect monads and semantics of effect systems. In: Jagannathan, S., Sewell, P. (eds.) The 41st Annual ACM SIGPLAN-SIGACT Symposium on Principles of Programming Languages, POPL 2014, San Diego, CA, USA, 20-21 January 2014, pp. 633-646. ACM (2014)

14. Lago, U.D., Gaboardi, M.: Linear dependent types and relative completeness. In: 2011 IEEE 26th Annual Symposium on Logic in Computer Science, pp. 133-142, June 2011

15. Laplaza, M.L.: Coherence for distributivity. In: Kelly, G.M., Laplaza, M., Lewis, G., Mac Lane, S. (eds.) Coherence in Categories. LNM, vol. 281, pp. 29-65. Springer, Heidelberg (1972). https://doi.org/10.1007/BFb0059555

16. Mac Lane, S.: Categories for the Working Mathematician. GTM, vol. 5. Springer, New York (1978). https://doi.org/10.1007/978-1-4757-4721-8

17. Melliès, P.-A.: Categorical semantics of linear logic. In: Interactive Models of Computation and Program Behaviour, vol. 27. Panoramas et synthses. Société Mathématique de France (2009)

18. Melliès, P.-A.: The parametric continuation monad. Math. Struct. Comput. Sci. 27(5), 651-680 (2017)

19. Milius, S., Pattinson, D., Schröder, L.: Generic trace semantics and graded monads. In: Moss, L.S., Sobocinski, P. (eds.) 6th Conference on Algebra and Coalgebra in Computer Science, CALCO 2015, 24-26 June 2015, Nijmegen, The Netherlands, vol. 35. LIPIcs, pp. 253-269. Schloss Dagstuhl - Leibniz-Zentrum fuer Informatik (2015) 
20. Petricek, T., Orchard, D., Mycroft, A.: Coeffects: unified static analysis of contextdependence. In: Fomin, F.V., Freivalds, R., Kwiatkowska, M., Peleg, D. (eds.) ICALP 2013. LNCS, vol. 7966, pp. 385-397. Springer, Heidelberg (2013). https:// doi.org/10.1007/978-3-642-39212-2_35

21. Reed, J., Pierce, B.C.: Distance makes the types grow stronger: a calculus for differential privacy. In: Hudak, P., Weirich, S. (eds.) Proceeding of the 15th ACM SIGPLAN International Conference on Functional Programming, ICFP 2010, Baltimore, Maryland, USA, 27-29 September 2010, pp. 157-168. ACM (2010)

22. Sato, T.: Approximate relational Hoare logic for continuous random samplings. Electr. Notes Theor. Comput. Sci. 325, 277-298 (2016)

23. Schöpp, U.: Computation-by-interaction with effects. In: Yang, H. (ed.) APLAS 2011. LNCS, vol. 7078, pp. 305-321. Springer, Heidelberg (2011). https://doi.org/ 10.1007/978-3-642-25318-8_23

24. Shao, Z. (ed.): ESOP 2014. LNCS, vol. 8410. Springer, Heidelberg (2014). https:// doi.org/10.1007/978-3-642-54833-8

25. Smirnov, A.L.: Graded monads and rings of polynomials. J. Math. Sci. 151(3), 3032-3051 (2008)

26. Street, R.: Two constructions on lax functors. Cahiers de Topologie et Géométrie Différentielle Catégoriques (1972)

Open Access This chapter is licensed under the terms of the Creative Commons Attribution 4.0 International License (http://creativecommons.org/licenses/by/4.0/), which permits use, sharing, adaptation, distribution and reproduction in any medium or format, as long as you give appropriate credit to the original author(s) and the source, provide a link to the Creative Commons license and indicate if changes were made.

The images or other third party material in this chapter are included in the chapter's Creative Commons license, unless indicated otherwise in a credit line to the material. If material is not included in the chapter's Creative Commons license and your intended use is not permitted by statutory regulation or exceeds the permitted use, you will need to obtain permission directly from the copyright holder. 